\title{
¡Ponga atención en los obstáculos epistémicos de los estudiantes! Una clave para enseñar derecho probatorio
}

\author{
Claudio Agüero San Juan* \\ Rodrigo Coloma Correa**
}

\begin{abstract}
ReSUMeN
Este artículo propone enseñar la competencia "resuelve conflictos jurídicos" en un curso de derecho probatorio usando problemas, en lugar de preguntas o casos. Los problemas son artefactos didácticos que se caracterizan porque, por un lado, fuerzan a los estudiantes a desarrollar la babilidad de producir, organizar y valorar pruebas que confirmen o refuten hechos relevantes en contextos análogos al judicial y, por el otro, permiten al profesor conocer las trayectorias de pensamiento de los estudiantes, hacer visibles sus obstáculos epistémicos y modificar sus rutinas de pensamiento. El análisis pone especial énfasis en el concepto de obstáculo epistémico y sus dificultades.
\end{abstract}

Derecho probatorio - formación inicial de abogados - obstáculos epistémicos

\section{Pay attention to the students' epistemic barriers. A key for teaching Evidence Law}

\begin{abstract}
This essay proposes to teach the competence of "solving legal conflicts" in an undergraduate Evidence Law module using "legal problems", instead of cases or questions. Legal problems are didactic devices characterized by two elements. First, students are compelled to develop the
\end{abstract}

* Licenciado en Derecho, Universidad Católica de Temuco. Doctor en Ciencias Humanas, Universidad Austral de Chile. Profesor de Derecho Constitucional, Universidad Alberto Hurtado. Correo electrónico: aguero. claudio@gmail.com

** Licenciado en Derecho, Pontificia Universidad Católica de Chile. Doctor en Derecho, Universidad Carlos III de Madrid, España. Profesor del Departamento de Ciencias del Derecho, Universidad Alberto Hurtado. Correo electrónico: rcoloma@uahurtado.cl

Este trabajo presenta resultados del proyecto financiado por el Fondo Nacional de Desarrollo Científico y Tecnológico, FONDECYT 1170872 "Prueba de los hechos. Coordinación entre el lenguaje de la teoría y el lenguaje de la práctica”. Ambos autores son investigadores de dicho proyecto.

Artículo recibido el 15 de agosto de 2018 y aceptado para su publicación en este número el 1 de agosto de 2019. 
skills to produce, organize and assess evidences that confirm or refute relevant facts in contexts analogous to judicial. Second, teachers are allowed to know the line of thought of their students, and consequently, by making epistemic barriers visible, to modify the student's thinking routines. The analysis places special emphasis on the concept of epistemic barrier and its difficulties.

Evidence Law - lawyers' initial training - epistemic barriers

\section{INTRODUCCIÓN}

$\mathrm{E}$ ste texto propone una metodología de trabajo para un problema pedagógico que es difícil de resolver en un curso de derecho probatorio. El asunto consiste en que los estudiantes tienden a transferir automáticamente sus prácticas de producción, organización y valoración de pruebas desde la cotidianeidad ${ }^{1}$ hacia los contextos jurídicos, lo que -en ocasiones- conduce a resultados deficientes. El déficit al que nos referimos se asocia a un despilfarro de las posibilidades explicativas disponibles desde el material probatorio disponible en un juicio y de las generalizaciones válidas, según lo estipulado por el sistema jurídico respectivo y lo concordado por una comunidad de especialistas. Aquello se hace visible fundamentalmente en el tipo de alegatos y sentencias que serán capaces de producir si asumen los roles de abogados y jueces ${ }^{2}$.

Todo proceso de aprendizaje aspira a que quienes hoy son estudiantes operen, en el futuro, de una manera "tecnologizada". Esta expectativa es difícil de satisfacer si no se erradican, de modo temprano, las formas de actuar propias del sentido común. El asunto, en palabras sencillas, es el siguiente. En un curso de derecho probatorio se espera que los estudiantes sean capaces de hacer ciertas cosas con pruebas, conjeturas y discursos justificativos. Ya en la primera clase del curso cualquier estudiante es capaz de resolver problemas sencillos de producción, organización y valoración de pruebas recurriendo a sus concepciones y rutinas previas ${ }^{3}$. Aunque hay sofisticadas "tecnologías de razonamiento probatorio" 4

${ }^{1}$ A esta afirmación subyace una tesis fuerte: las personas producimos, organizamos y valoramos pruebas a diario y este razonamiento probatorio cotidiano es común a todos los participantes de una comunidad. Este modo de valorar hechos nos resulta útil en tareas simples como elegir un regalo para nuestra madre, decidir cómo armar una maleta para las vacaciones o formarnos una opinión acerca de si un político miente en una conferencia de prensa. Asumimos la verdad de esta tesis como un postulado, pues su análisis excede los límites de este trabajo.

${ }^{2}$ De la manera de hacerse cargo de lo probatorio en las sentencias, ver Accatino, Daniela, "La arquitectura de la motivación de las premisas fácticas de las sentencias judiciales”, en PÁEz, Andrés, Hechos, evidencia y estándares de prueba, Universidad de los Andes, Bogotá, 2015, pp. 65 y ss.

${ }^{3}$ La expresión "concepciones previas" hace referencia a un conjunto heterogéneo y difuso de saberes y nociones culturales de los estudiantes. Las concepciones previas de los estudiantes concernientes a la prueba "son creencias muy arraigadas sobre cómo funciona el mundo y esas creencias sobreviven a pesar de la enseñanza formal y a menudo coexisten con las ideas 'científicas'”. William, Leonard; Gerace, William y Dufresne, Robert, "Resolución de problemas basada en el análisis. Hacer del análisis y del razonamiento el foco de la enseñanza de la física", en Enseñanza de las Ciencias, No 20 (3), 2002, p. 389.

${ }^{4}$ La palabra "tecnología" es una metáfora que enfatiza que la comunidad jurídica no acepta cualquier estructura discursiva, ni forma de valorar pruebas, ni tipo de razonamiento. Cfr. García Petit, María Cecilia y 
disponibles $^{5}$, es curioso que la mayoría de las respuestas iniciales de los estudiantes puedan resultar satisfactorias para variados contextos legos como lo sería también un razonamiento periodístico respecto de un caso judicial bullado ${ }^{6}$. Sin embargo, esas mismas respuestas serán claramente insuficientes cuando se ubican en contextos especializados como lo es la judicatura. Así como el estudiante no conoce las formas más sofisticadas de operar con pruebas, seguramente no advertirá qué distingue el razonamiento probatorio cotidiano del razonamiento probatorio especializado. Para mejorar su desempeño el estudiante se esforzará, entonces, por poner un poco más de cuidado en lo que hace (siempre) desde el sentido común, pero no advertirá que la trayectoria de todo lo que hace debiera ser diferente.

La enseñanza del derecho probatorio no es útil si se subordina totalmente a disposiciones normativas vigentes ${ }^{7}$. Esta afirmación se funda en dos tipos de razones. En primer lugar, en el plano del diseño institucional, progresivamente las disposiciones que hacen referencia al resultado probatorio han sido desplazadas por disposiciones minimalistas que aseguran la vigencia de un sistema de Sana $\mathrm{Crítica}^{8}$. Así, la densidad normativa del derecho probatorio es cada vez menor. En segundo lugar, en un plano epistemológico, la racionalidad del razonamiento probatorio en contextos jurídicos - a diferencia del razonamiento lógicomatemático- no depende únicamente del seguimiento de reglas explícitamente formuladas. Un razonamiento probatorio sofisticado ha domesticado un variado conjunto de razones para creer: conocimientos científicos, percepción directa de los fenómenos, testimonios fiables, etcétera. En este plano, buena parte de la racionalidad del razonamiento probatorio en contextos jurídicos no depende de lo que dice la ley.

En este contexto, creemos que la enseñanza del derecho probatorio vale la pena si asume un cierto grado de complejidad y se ocupa de fortalecer competencias tales como "resuelve conflictos jurídicos en contextos de actuación profesional" 9 . Para lograr lo recién anunciado es necesario identificar lo que funciona mal en el proceso de resolución de un

AgüEro SAn JuAn, Claudio, "Bases para el estudio de la dinámica discursiva en la comunidad jurídica chilena”, Rev. Derecho (Valdivia). 2014, vol. 27, n. 1, pp. 59-79.

${ }^{5}$ Anderson, Terence; Schum, David y Twining, William, Análisis de la prueba, Marcial Pons, Madrid, 2015, pp. 153-204.

${ }^{6}$ Nótese que el trabajo de los periodistas también debe gestionar medios de prueba (llamados fuentes periodísticas en la jerga especializada) para construir la información que comunica.

${ }^{7}$ Esto fue observado por autores de la talla de Bentham, Thayer y Wigmore. No es de extrañar, entonces, que en el siglo XX, en el ámbito anglosajón, se haya producido un cambio en la clase de artículos publicados. Cfr. PArk, R., Saks, M., "Evidence Scholarship Reconsidered: Results of the Interdisciplinary Turn”, en 47 Boston College Law Review, 2006, p. 952.

${ }^{8}$ Agüero San JuAn, Claudio. "La Sana Crítica como cláusula general”, en Benfeld, Johann y Larroucau, Jorge (editores), La sana crítica bajo sospecha, publicaciones de la Escuela de Derecho PUCV, Valparaíso, 2018, p. $153-162$.

9 Técnicamente, la competencia es más compleja. En ocasiones se espera de los abogados o jueces la gestión de un problema, sin que necesariamente se llegue a una solución, entendida en términos fuertes. Para los efectos de no apartarnos de la terminología estándar hablaremos de esta competencia usando solo el término "resuelve". 
conflicto, explicitar los pasos a seguir para su resolución y, a la vez, modificar las rutinas de pensamiento que optimicen la producción de soluciones conformes con el derecho ${ }^{10}$.

El uso de problemas como método que guía la interacción entre profesores y estudiantes hace posible la detección de fallos en la manera de actuar de los estudiantes. A estos fallos metodológicos los llamaremos obstáculos epistémicos ${ }^{11}$. La terminología proviene del filósofo de la ciencia francés Gastón Bachelard, quien trabajó el concepto en su La formación del espíritu científico: contribución a un psicoanálisis del conocimiento. En ese libro Bachelard señala: "En el acto mismo de conocer, íntimamente, por una suerte de necesidad funcional, aparecen morosidades y trastornos [...], causas de estancamiento e incluso de regresión [...] que llamaremos obstáculos epistemológicos [...] Detectar los obstáculos epistemológicos contribuye a fundar los rudimentos de un psicoanálisis de la razón" ${ }^{2}$.

La identificación y superación de los obstáculos epistémicos que afectan la resolución de un problema no es una tarea sencilla, tanto es así que no es inimaginable que un estudiante logre avanzar en el dominio de la competencia sin llegar a modificarlos por completo ${ }^{13}$. Es por ello que, probablemente, la mejor manera de representar el dominio de la competencia de resolución de conflictos jurídicos sea una espiral. Las espirales carecen de fronteras nítidas en sus distintos niveles y admiten diferentes puntos de observación. Los estudiantes ascienden en el nivel de dominio de la competencia así como una persona sube por una escalera de caracol, donde no siempre es claro en qué piso se encuentra. Así las dificultades de un futuro abogado pueden situarse en distintos puntos, los que dan cuenta de diferentes obstáculos epistémicos ${ }^{14}$ que exigen detenerse para trabajar respecto de ellos modificando rutinas de pensamiento y acción.

Los proyectos orientados al fortalecimiento de la competencia "resuelve conflictos jurídicos" saldrán airosos en la medida de que quienes logran dominar ciertos saberes y estrategias se inserten mejor en el campo de trabajo de los juristas, que quienes no lo

${ }^{10}$ Esta competencia expresa una paradoja. Por un lado, los abogados son una comunidad profesional con estándares de desempeño y técnicas de evaluación de rendimiento y, al mismo tiempo, la formación de los abogados es cada vez más desprofesionalizada, porque empleadores y universidades enfatizan la enseñanza de competencias menos técnicas. Este problema no es exclusivo del derecho. En medicina ocurre algo semejante. Cfr. Hernández, Juan, Sociología de las profesiones sanitarias, Escuela Nacional de Sanidad, Madrid, 2012, Tema 6.9.

${ }^{11}$ Los obstáculos epistémicos son comunes a toda disciplina científica. En derecho, ellos afectan las actividades de creación, aplicación y ejecución del derecho. Dentro de la aplicación del derecho, sospechamos que es urgente considerarlos al abordar el razonamiento probatorio.

${ }^{12}$ BaCHelard, Gastón, La formación del espíritu científico: contribución a un psicoanálisis del conocimiento objetivo, Siglo XXI, Madrid, pp. 13-19.

13 Comprender el rol de los obstáculos en el pensamiento y las razones de su pervivencia (a pesar de la enseñanza explícita) requiere considerar que integran una red de elementos (creencias, experiencias previas, valoraciones, emociones) que se refuerzan entre sí. MARzÁbal, Ainoa; Merino, Cristián; Rocha, Alejandro, "El obstáculo epistemológico como objeto de reflexión para la activación del cambio didáctico en docentes de ciencias en ejercicio", en Revista electrónica de investigación en educación en ciencias REIEC, vol. 9. № 1, 2013, pp. 70-83.

${ }^{14}$ Las dificultades para formular soluciones plausibles también es consecuencia de déficits en conocimientos disciplinarios. Aun cuando los problemas son una buena herramienta para identificar esas carencias no es su uso más eficiente. 
hayan logrado. El asunto merece atención. Si los abogados y jueces no son capaces de optimizar los recursos probatorios (pruebas y argumentos) y solo se limitan a hacer más o menos lo que cualquier persona medianamente culta se encuentra en condiciones de realizar, es previsible que en un mediano plazo pierdan la exclusividad en la gestión de los problemas de prueba en los procesos judiciales ${ }^{15}$.

\section{I. ¿Qué es un OBSTÁ́culo EPISTÉmico?}

No es inusual que en la fase inicial de un aprendizaje cualquiera -jugar tenis, dibujar figuras humanas o tocar el clarinete- el maestro pida dejar de lado todo lo que el estudiante cree saber acerca de la respectiva práctica. La petición puede ser frustrante porque el estudiante debe desaprender lo que ya cree saber o el modo en que ejecuta ciertos procedimientos ${ }^{16}$. La incomodidad inicial desaparecerá solo cuando se arribe a un alto nivel de desempeño que permita hacer un autoexamen de la práctica anterior. Aquello que sabemos en un contexto de principiantes, pero que dificulta el progreso cuando se trata de alcanzar un desempeño avanzado, es lo que llamaremos obstáculo epistémico ${ }^{17}$.

Un obstáculo epistémico es un conocimiento o una forma de actuar eficaz para legos, pero inadecuado para especialistas ${ }^{18}$. En ese sentido, propenden a la economía del proceso mental ${ }^{19}$. Es más fácil creer que el Sol gira en torno a la Tierra, porque lo vemos salir y ocultarse por el horizonte, que creer que la Tierra rota sobre sí misma, aunque no podemos percibir su movimiento. Este ejemplo permite apreciar que los obstáculos se

${ }^{15}$ Coloma, Rodrigo, "Las disciplinas jurídicas y su reinvención”, en Ius et Praxis, año 22, No 2, 2016, pp. 253-298.

${ }^{16}$ Por ejemplo, es posible que una cierta manera de tomar la raqueta, el lápiz o el instrumento puede ser eficiente para lograr un dominio básico, pero puede ser una traba para llegar a un dominio avanzado.

${ }^{17}$ En la enseñanza de la física un obstáculo de este tipo es la asociación intuitiva entre fuerza y movimiento. Ese vínculo impide apreciar que un péndulo estático - a pesar de que no se mueve- está sometido a la fuerza de la gravedad. Marzábal, Ainoa; Merino, Cristián; Rocha, Alejandro, "El obstáculo epistemológico como objeto de reflexión para la activación del cambio didáctico en docentes de ciencias en ejercicio", en Revista electrónica de investigación en educación en ciencias REIEC, vol. 9. N 1 1, 2013, pp. 70-83. Otros textos que tratan de los obstáculos epistémicos son: Mora, Arabela, "Obstáculos epistemológicos que afectan el proceso de construcción de conceptos del área de ciencias en niños de edad escolar", en InterSedes, Revista de las Sedes regionales, Universidad de Costa Rica, vol. 3, No 5, mayo 2002, pp. 75-89. Astolfi, Jean Pierry, "El trabajo didáctico de los obstáculos, en el corazón de los aprendizajes científicos”, en Enseñanza de las Ciencias, vol. 12, No 2, 1994, pp. 206-216. AsTOLFI, Jean Pierry, El error un medio para enseñar, Díada, Sevilla, 2011, 100 páginas. Astolfi, Jean Pierry y Peterfalvi, Brigitte, "Estrategias para trabajar los obstáculos: dispositivos y resortes", en Camilloni, Alicia (comp.), Los obstáculos epistemológicos en la enseñanza, Gedisa, Barcelona, 1997, 224 páginas.

${ }^{18}$ El error manifiesta un conocimiento anterior que, a pesar de su éxito pasado, es inadecuado para resolver una tarea conforme con patrones de actuación de una disciplina. Brousseau, G., Théorie des situations didactiques, La Pensée Sauvage, Grenoble, 1998.

${ }^{19}$ La utilidad en situaciones escasamente exigentes hace dudosa la conveniencia del uso de la locución "obstáculo" (porque comunica dificultades). Su uso en la reflexión educativa y la ambigüedad que presentan palabras como "heurística" nos han inclinado a usarla pese a la objeción recién señalada. 
articulan en una red de creencias intuitivas y en emociones ${ }^{20}$, y que por ello son difíciles de desarraigar, pues han sido eficaces para afrontar la vida cotidiana. Así, a pesar de su fracaso en contextos especializados, es corriente que evitemos desecharlos ${ }^{21}$. De hecho, día a día, seguimos usando expresiones tales como "puesta de sol" y "hora de salida del sol” aun cuando sabemos hace siglos su falsedad astronómica.

El derecho probatorio es un saber estratégico, esto quiere decir que debe aprenderse en un procedimiento de reflexión más o menos artificial que fuerza la optimización de la información disponible conforme ciertos objetivos ( $v . g r$. el interés del cliente, obtener una decisión judicial, etcétera $)^{22}$. Este modo de entender el derecho probatorio nos permite ensayar una definición de obstáculo epistémico en este sector del derecho. Sostenemos que los obstáculos epistémicos del derecho probatorio son formas de gestionar información que se ejecutan en la experiencia cotidiana por sujetos carentes de formación especializada en técnicas de prueba y de decisión de hechos (legos). Estas formas de gestionar datos son trasladadas mediante la analogía a contextos especializados de gestión de información como un proceso judicial y ahí se constituyen en impedimentos para lograr un desempeño satisfactorio $^{23}$. Veamos un ejemplo. Las interacciones en la vida cotidiana están fundadas en la confianza. Así, el estándar de prueba que usamos para validar lo que se nos dice depende del contexto, de las personas involucradas y de nuestra mayor o menor disposición a usar información provista por terceros como piezas de nuestros discursos ${ }^{24}$, entre otras variables $^{25}$. Podemos calificar a este estándar como folk en la medida en que es usado en la vida

${ }^{20}$ Festinger usa la expresión reducción de la disonancia cognitiva para llamar al modo en que disminuimos la inconsistencia entre nuestras creencias y acciones. El modo de evitar la incomodidad es usar razones (excusas o justificaciones) que rechazan la información nueva que incrementa la disconformidad con las creencias iniciales. Festinger, León, Teoría de la disonancia cognoscitiva, Instituto de Estudios Políticos, Madrid, 1975, pp. 14-16.

${ }^{21}$ La idea de obstáculo epistémico se puede vincular con los trabajos de Tversky y Kahneman acerca de sesgos en la toma de decisiones bajo incertidumbre. Sus textos más conocidos dan cuenta del uso de ciertas formas de razonamiento muy sencillos (heurísticas) que -en muchas oportunidades-conducen a resultados inconsistentes con el cálculo probabilístico. Las heurísticas -que son una forma de obstáculo epistémico- que ellos identifican resultan fáciles de visualizar en la vida diaria y creemos que pueden transferirse al razonamiento jurídico. La conciencia de estas heurísticas es clave para fijar medidas correctivas. Ver TvERSKY, Amos y KaHNEMAN, Daniel, “Judgment Under Uncertainty: Heuristics and Biases”, en Science, vol. 185, 1974. Hay versión castellana disponible: Tversky, Amos y Kahneman, Daniel, "El juicio bajo incertidumbre: heurísticas y sesgos”, en Kahneman, Daniel, Pensar rápido, pensar despacio, Debate, Buenos Aires: Debate, 2012, pp. 545-567.

${ }^{22}$ En este sentido, es deseable contar con modelos más explícitos acerca de qué es lo que debe hacerse para una gestión de pruebas y decisión de hechos satisfactoria. Ver Anderson, Terence; ScHum, David y Twining, William, Análisis de la prueba, Marcial Pons, Madrid, 2015; Coloma, Rodrigo, "Bases conceptuales de un modelo para decidir hechos”, en Doxa Cuadernos de Filosofía del Derecho, No 40, 2017, pp. 69-92.

${ }^{23}$ Nos referimos con ello a las principales tareas a cargo de abogados y jueces, respectivamente, en lo que atañe a asuntos de prueba en los procesos judiciales.

${ }^{24}$ Ver González Lagier, Daniel, "Distinciones, estipulaciones y sospechas sobre los criterios de valoración y los estándares de prueba”, en BENFELD, Johann y LARRouCAU, Jorge (eds.), La sana crítica bajo sospecha, Ediciones Universitarias de Valparaíso, Valparaíso, 2018, pp. 23-24.

${ }^{25}$ En la emisión radiofónica de la Guerra de los Mundos que realizó Orson Wells en 1938 el efecto de verosimilitud en los oyentes radica en la estructura discursiva de un programa de noticias y no en el contenido de lo narrado. 
cotidiana con relativo éxito ${ }^{26}$. No es raro que aceptemos una afirmación como verdadera solo porque nuestra intuición nos lo sugiere. Si en estos casos somos interrogados respecto de nuestra disposición a creer, tal vez agreguemos a nuestro discurso justificativo algunas circunstancias (conocer al interlocutor, experiencias previas acerca de la verdad de lo que él dice, entre otras variables). Un elemento clave para determinar la aceptación de lo que se nos dice es nuestra propia disposición a usarlo en discursos posteriores sin formular prevenciones respecto de qué podría no ser verdadero e, incluso, a omitir nuestra fuente de información ${ }^{27}$.

La convergencia de algunos rasgos de credibilidad resulta suficiente para dar por verdadero lo que se está afirmando. Este estándar folk, sin embargo, no debería ser usado para resolver un problema probatorio en el contexto judicial como lo es un juicio penal, porque tal procedimiento exige que el acto típico y la participación del acusado deben probarse con el estándar "más allá de toda duda razonable" 28.

En las simulaciones de juicio oral hemos observado que los estudiantes tienen tendencia a usar el estándar folk. Esa tendencia es la manifestación de un obstáculo epistémico: creer que un juez debe razonar ante los hechos del mismo modo que una persona sin formación jurídica especializada. Incluso ante un esfuerzo consciente, es común que los estudiantes usen el estándar folk y, entonces, que no tengan en cuenta, por ejemplo, las posibilidades de producción de prueba de la situación en concreto $^{29}$, el modo en que deben sortear la posibilidad de explicaciones alternativas ${ }^{30}$, las restricciones desde la ética profesional, las reglas de producción de prueba en el proceso penal y varios otros asuntos por el estilo.

\section{II. ¿QUÉ ES UN PROBLEMA PROBATORIO?}

Usamos la expresión "problema” en reemplazo de la palabra "caso" por defectos semánticos asociados a esta última. Un problema es un artefacto didáctico que pone en

${ }^{26}$ Las investigaciones sobre noticias falsas (fake news) distribuidas por redes sociales prueban que los lectores comunes tienen bajos estándares para discriminar lo que es verídico y confiable de aquello que no lo es. Cfr. White, Aidan, "Información falsa: La opinión de los periodistas", en Correo de la Unesco. Disponible en "https://es.unesco.org/courier/2017-julio-septiembre/informacion-falsa-opinion-periodistas" [última visita: 16 de mayo 2018].

$27 \mathrm{Si}$, por ejemplo, una amiga nos dijese: "Jorge está viviendo en las islas Galápagos", evaluaremos rápidamente el contexto en que se nos comunica la afirmación, el historial de nuestra amiga y la actitud que adopta mientras nos habla. Si nosotros, a su vez, contamos seriamente a un tercero "Jorge está viviendo en las islas Galápagos” sin añadir información alguna, es porque nuestro estándar folk ha sido superado.

${ }^{28}$ Usando otro marco conceptual este problema lo aborda Laudan en LAUDAN, Larry, "Por qué un estándar de prueba subjetivo y ambiguo no es un estándar", en Doxa Cuadernos de Filosofía del Derecho, No 28, 2005. La dimensión subjetiva (confianza) involucrada en el estándar folk, impediría, incluso, sostener que se está usando un estándar de prueba.

${ }^{29}$ Coloma, Rodrigo, "Estándares de prueba y juicios por violaciones a los derechos humanos", en Revista de Derecho Universidad Austral de Chile, vol. XXII, No 2, 2009, pp. 220 y ss.

${ }^{30}$ Ferrer, Jordi, La valoración racional de la prueba, Marcial Pons, Madrid, 2007, p. 147. Laudan, Larry, "Por qué un estándar de prueba subjetivo y ambiguo no es un estándar", en Doxa Cuadernos de Filosofía del Derecho, No 28, 2005, p. 107. 
evidencia uno o varios errores en el pensamiento de los estudiantes. Sin perjuicio de que la experiencia propia o ajena es útil para la formulación de un problema, es importante aclarar que el problema no es descubierto, ni reciclado de la práctica profesional, sino pensado específicamente para un grupo de estudiantes con una finalidad específica: superar un obstáculo epistémico previamente identificado. Un “caso”, en cambio, es un artefacto didáctico que comunica una interrogante real o ficticia que se usa para que los estudiantes argumenten pertinente a la respuesta preferible ${ }^{31}$. El "caso" suele poner de relieve que el supuesto de hecho no tiene una única respuesta correcta dentro del sistema de las fuentes y que es plausible construir varias respuestas igualmente aceptables a partir de los materiales jurídicos disponibles.

Un ejemplo de problema sería el siguiente: "En julio de 1994, el Estado de California acusó a Orenthal James Simpson (OJS) de asesinar a su exesposa, Nicole Brown Simpson (NBS), y a un tercero, Ronald L. Goldman (RLG), el 12 de junio de 1994 [.... Uno de los asuntos era si OJS podría haber tenido la oportunidad de cometer los crímenes. La única prueba presentada en el juicio, relacionada con ese punto, es resumida a continuación. En algún momento entre las 10:15 y las 10:20 p.m., el 12 de junio de 1994, Pablo Fuentes escuchó el "aullido quejumbroso" de un perro. Alrededor de las 10:45 p.m., Steven Schwab encontró al perro Akita de NBS con sangre en sus patas. Aproximadamente a la misma hora, Kato Kaelin, un invitado en la casa de OJS, escuchó tres golpes en la pared [...] 1. Construya un relato que dé cuenta de cada uno de los eventos y observaciones detallados más arriba, mostrando que OJS casi con toda seguridad pudo haber asesinado a NBS y RLG. 2. Construya un relato que dé cuenta de cada uno de los eventos y observaciones detallados más arriba, mostrando que OJS casi con toda seguridad no pudo haber asesinado a NBS y RLG"32.

El estudiante para construir una respuesta deberá elegir una estrategia, según esta, asumirá determinados riesgos indicativos de una mayor o menor adhesión a los datos que se le presentan ${ }^{33}$. El profesor, así, dispondrá de diferentes indicios acerca de lo que dificulta que el estudiante optimice el material probatorio disponible recurriendo a generalizaciones aceptadas en el contexto de los procesos judiciales; es decir, podrá advertir obstáculos epistémicos que afectan la argumentación ${ }^{34}$.

${ }^{31}$ Un ejemplo de caso es el siguiente: ¿Conforme al derecho chileno, es posible que una mujer arriende su útero? La pregunta se formula con el propósito de que los estudiantes construyan un discurso que justifique una solución afirmativa o negativa conforme con el sistema de fuentes del derecho vigente. En el derecho chileno, diferentes casos y preguntas pueden encontrarse, por ejemplo, en el libro de FiguEROA, Gonzalo. Curso de Derecho Civil: Materiales para clases activas. Teoría de la ley los derechos subjetivos. Editorial Jurídica de Chile, segunda edición, 1988.

32 Anderson, Terence; Schum, David y Twining, William, Análisis de la prueba, Marcial Pons, Madrid, 2015 , pp. 56-57.

${ }^{33}$ El estudiante podrá, por ejemplo, introducir nueva información que fortalezca la coherencia del relato, pero que comunica un bajo compromiso con la información que se le entrega. Otra opción será, por ejemplo, ceñirse a la información disponible y abusar de conectores como "entonces", "por lo tanto" y otros semejantes, generando una apariencia de rigor analítico, aun cuando nada agregue al texto en el que se trabaja.

${ }^{34}$ A ello hay que agregar que la locución "con toda seguridad” es útil para detectar obstáculos epistémicos relacionados con el uso de los estándares de prueba. 
Un buen problema es creado para que los estudiantes, al relacionar la información existente con ciertas inferencias, se enfrenten a una encrucijada: usar la rutina de pensamiento folk o el modo especializado de arribar a la solución. En la literatura educacional los problemas pueden clasificarse según diversos criterios. Así, por ejemplo, pueden ordenarse según si el énfasis está puesto en aprendizajes conceptuales o en aprendizajes metodológicos, procedimentales o estratégicos. Profundizaremos solo en este último tipo de problema que, a su vez, puede ser subclasificado en problemas de investigación, de comunicación y de innovación ${ }^{35}$.

(1) Problemas que desarrollan habilidades de investigación. Estas habilidades son las siguientes: conocimiento de la causa del problema jurídico; análisis de las partes del problema; establecimiento de conjeturas contrastables; identificación de relaciones entre variables del problema; planteamiento de una estrategia de resolución de un problema; descripción de situaciones fácticas; identificación de propiedades, características o requisitos; utilización de criterios de clasificación de normas, hechos, actos, contratos o instituciones jurídicas; elaboración de generalizaciones o conclusiones (fácticas o normativas); emisión de juicios fundados respecto de la calidad del procedimiento seguido o de sus resultados, entre otras.

(2) Problemas que desarrollan competencias de comunicación predefinidas o estereotipadas. La solución de estos problemas requiere escribir géneros jurídicos típicos (demandas, recursos, contratos, minutas de alegato, etc. $)^{36}$.

(3) Problemas que exigen innovar usando un conjunto finito de materiales jurídicos disponibles. Estos problemas desarrollan la capacidad de adaptar y adecuar los conocimientos en el derecho vigente para formular una solución ad hoc. Esta exigencia ocurre cuando se pide, por ejemplo, usar las normas existentes para satisfacer una especial combinación de necesidades o intereses, ya sea institucionalizándolos (como en una serie de contratos vinculados) o planteando vías para su protección y tutela (formulando el modo más efectivo para impugnar una decisión administrativa).

Los problemas del tipo (1) requieren estructurar un discurso ordenado del tópico pedido y no solo exigen identificar la solución que fija la dogmática dominante o la interpretación estándar de una ley. En cambio, en los problemas del tipo (2) hay que formular un texto profesional de la comunidad jurídica ${ }^{37}$.

En relación con los problemas del tipo (3) y sus diferencias con los problemas de tipo (1) es preciso señalar que los primeros buscan una solución jurídica justificada. Esto, por regla general, implica dos tareas: (a) formular una interpretación correctora de las fuentes formales vigentes para resguardar ciertos intereses presentando una solución "nueva" dentro

${ }^{35}$ Estos tres tipos de problemas pueden integrarse entre sí cuando se formula un problema complejo.

${ }^{36}$ La principal dificultad es elaborar un texto con todas las exigencias formales y de fondo que rigen en la comunidad jurídica de referencia. En palabras de Carlino "enseñar géneros académicos es, entonces, posibilitar que los alumnos se incluyan en situaciones discursivas típicas de comunidades especializadas, según propósitos, significados y valores compartidos”. Cfr. CARlino, P., “Alfabetización académica diez años después”, en Revista Mexicana de Investigación Educativa, vol. 18 (57), 2013, p. 362.

${ }^{37}$ El uso de estos problemas no es usual, porque los profesores suponen que los procedimientos de escritura se aprenden en los primeros empleos por imitación o por participación en la enseñanza habitual y, entonces, no hay actividades de enseñanza explícitas en el plan de estudios. 
de una teoría o conjunto de afirmaciones que es parte dogmática y parte filosofía ${ }^{38}$, y (b), justificar esa solución como conforme al derecho, según esa doctrina dogmática o teoría filosófica.

Muchos profesores no construyen problemas, sino que trasladan tareas desde su ámbito profesional. Los profesores también modifican esas tareas para hacerlas más simples o complejas. En ambos supuestos, estos pseudoproblemas no se ajustan a las necesidades de los estudiantes y no desarrollan las competencias que el currículum compromete ${ }^{39}$.

Los problemas para el curso de derecho probatorio son complejos, porque integran competencias de investigación, de comunicación y de innovación. Un buen problema de derecho probatorio permite: (a) desarrollar el razonamiento jurídico en situaciones funcionales y no solo exponer cuál es la solución estimada correcta dentro de una posición dogmática o conjunto de saberes teóricos; (b) descubrir, recolectar, organizar y estructurar datos de situaciones reales y no solo interpretar textos jurídicos, y (c), elaborar generalizaciones acerca de hechos que la formulación del problema no explicita.

\section{III. ¿Qué OBSTÁCUlOS EPISTÉMICOS SON RELEVANTES EN UN CURSO DE DERECHO PROBATORIO?}

Esta sección se ocupa de la identificación de algunos obstáculos epistémicos relevantes para la didáctica de un curso de derecho probatorio. La planificación exige prestar atención a los conocimientos exigibles a los estudiantes, al rol del profesor y a los productos que servirán como evidencia de los aprendizajes.

(a) ¿Quéconocimientos se necesitan para fisurar un obstáculo epistémico dado? Dependiendo de la clase de obstáculos epistémicos de los que un curso de derecho probatorio se haga cargo, los estudiantes requerirán distintos niveles de dominio de teorías y conceptos dogmáticos. La superación de obstáculos epistémicos complejos supone dominar saberes teóricos y dogmáticos avanzados. Solo así es posible resolver problemas jurídicos difíciles. A contrario, para diagnosticar los obstáculos epistémicos básicos no es necesario pensar en problemas jurídicos: asuntos cotidianos y de ámbitos extrajurídicos son suficientes.

Aquí debe advertirse que un curso de derecho probatorio no necesariamente funcionará mejor si se ubica al final del plan de estudios. Lo único que podemos afirmar es que la ubicación del curso cambiará el tipo de obstáculo epistémicos que podrá ser abordado en él.

(b) ¿Cuál es el rol del profesor en la identificación de obstáculos epistémicos? Según Astolfi ${ }^{40}$, el profesor debe, en primer lugar, ayudar al estudiante a identificar el obstáculo. Esto implica

${ }^{38}$ El adjetivo "nuevo" es minimalista. No se trata de pedir al estudiante la creación ex nibilo de una solución. Se trata de pedir al estudiante la justificación de una solución no explícita dentro de las fuentes del derecho.

39 También hemos observado que los profesores diseñan problemas según sus propios obstáculos epistémicos en lugar de aquellos que afectan a los estudiantes.

${ }^{40}$ Ver los trabajos ya citados de Astolfi, Jean Pierry, "El trabajo didáctico de los obstáculos, en el corazón de los aprendizajes científicos", en Enseñanza de las Ciencias, vol. 12, No 2, 1994, pp. 206-216; Astolfi, Jean Pierry, El error un medio para enseñar, Díada, Sevilla, 2011, 100 páginas; y Astolfi, Jean Pierry y Peterfalvi, Brigitte, "Estrategias para trabajar los obstáculos: dispositivos y resortes", en CAmilloni, Alicia (comp), Los obstáculos epistemológicos en la enseñanza, Gedisa, Barcelona, 1997, 224 páginas. 
hacer evidente que es un tejido de ideas que permite interpretar la situación analizada con un resultado divergente del deseado ${ }^{41}$. En segundo lugar, el profesor debe desestabilizar el obstáculo, es decir, generar incoherencias en el sistema de creencias del estudiante para forzarlo a abandonar sus creencias iniciales. En tercer lugar, ayudarlo a trabajar con el conocimiento experto y a expresar sus pensamientos usando las nuevas herramientas (conceptos y procedimientos). De acuerdo con estas ideas, el profesor de un curso de derecho probatorio deberá facilitar la reflexión respecto del propio desempeño, es decir, retroalimentar los errores de los estudiantes dando apoyo en el proceso de pensar como abogado.

Es importante, eso sí, que las intervenciones del profesor no debiliten el problema a enfrentar. Los estudiantes progresivamente deberán ir ganando en autonomía y aquello supone también identificar dificultades (no solo resolverlas). Así, el estudiante debe entender que el trabajo desde el sentido común resulta insuficiente, habida cuenta de las expectativas que se ciernen respecto de jueces y abogados ${ }^{42}$.

(c) ¿Qué productos deberían construir los estudiantes para evidenciar eventuales obstáculos epistémicos? El listado de textos o discursos que deben elaborarse para demostrar un aprendizaje es clave. Todos sabemos que el desempeño profesional del abogado tiene una dimensión impredecible e incierta que es más relevante que su dimensión rutinaria. La formación práctica debe enfatizar que el estudiante tome decisiones en condiciones de incertidumbre. Esto implica asumir una actitud de indagación en orden a buscar, seleccionar e interpretar información ausente o incompleta o en orden a evaluar la posibilidad de desarrollar varias acciones profesionales alternativas para solucionar un problema jurídico.

\section{LA CONCIENCIA DE LOS OBSTÁCULOS EPISTÉMICOS COMO CONDICIÓN PARA ACTUAR COMO ABOGADOS EN CONTEXTOS PROBATORIOS}

A continuación presentamos algunos conceptos o prácticas en el derecho probatorio afectados por obstáculos epistémicos interrelacionados.

(a) Sobre "los hechos" y los "bechos probados". La noción de "hechos" propia del derecho probatorio asume que están cargados de dogmática. Esto quiere decir que los hechos probados que se fijan en los procesos judiciales dependen de los significados de las disposiciones aplicables. A los estudiantes les resulta difícil entender que los abogados no se interesan por todo lo que pasó, sino solo por aquello que es útil para los efectos de asignar un deber o un derecho. El obstáculo epistémico que impide aprehender este concepto está dado por una noción de hecho diferente, la que podría reconstruirse como sigue: los hechos probados

${ }^{41}$ Estas afirmaciones suponen que el plan de estudios organiza actividades de enseñanza y evaluación que vinculan teoría y práctica. Cfr. LuCARELLI, F., "Teoría y práctica como innovación en docencia, investigación y actualización pedagógica”, en Cuadernos de Investigación, $\mathrm{N}^{\circ} 10$, Instituto de Ciencias de la Educación, Buenos Aires, Facultad de Filosofía y Letras, UBA, 1999.

42 Acerca de las interacciones entre estudiantes y profesores Cfr. BurRIDGE, Roger, "Learning law and legal expertise by experience", en BuRRIDGE, Roger et al., Effective learning E teaching, The Institute for Learning and Teaching in Higher Education, Londres, 2002, pp. 25-51. 
son simétricos a los eventos ocurridos en el mundo y ambos tipos de hechos son accesibles sin la intermediación de teoría alguna. Este obstáculo se manifiesta en no menos de tres errores: (i) la reconstrucción de la narración se hace de una forma desconectada del derecho aplicable ${ }^{43}$, (ii) la reconstrucción del hecho abusa de la cronología y, (iii) la reconstrucción del hecho no prevé teorías del caso alternativas.

Un concepto de hecho útil sería el siguiente: hecho (probado) es un artefacto lingüístico que adscribe: (1) acciones u omisiones jurídicamente relevantes acerca de uno o más sujetos, (2) y que sometido a un proceso de comprobación/refutación (3) ha superado el estándar de prueba institucionalmente establecido ${ }^{44}$.

(b) Desarrollo de la actividad de valoración de la prueba. El modo en que un abogado realiza el proceso mental que llamamos "valoración de la prueba" es muy diferente del modo en que un lego realiza la misma tarea en la vida diaria. La reconstrucción del lego está regida por la coherencia de la narrativa. En cambio, la reconstrucción del abogado está orientada por la sustentación de un factum probandum ${ }^{45}$. De acuerdo con estas ideas el obstáculo epistémico que enfrentan los estudiantes puede ser descrito como un proceso de valoración de la prueba carente de rigor analítico, donde quien resuelve el problema se conforma con la construcción de un relato que no cuida de virtudes epistémicas importantes para el derecho ${ }^{46}$; como lo son el anclaje en la información que se produce en la audiencia de prueba, la completitud y coherencia de la estructura argumental y la economía del mensaje $\mathrm{e}^{47}$. Este obstáculo se manifiesta en varios errores: dejar cabos sueltos o vacíos que no pueden ser probados, el uso de generalizaciones, prejuicios, estereotipos o inferencias no explicitadas para soportar la valoración de la prueba, y no precaver la crítica de la contraparte. Así, el lego opera bajo supuestos como: (a) los testigos presenciales recuerdan fielmente lo ocurrido; (b) si el acusado usa su derecho a guardar silencio es porque oculta algo;

43 Agüero San Juan, Claudio. "Una crítica al modelo de la narración judicial como explicación científica", Ius et Praxis, 2014, vol. 20, n. 1, pp. 221-252.

${ }^{44}$ Coloma, Rodrigo, "Bases conceptuales de un modelo para decidir hechos", en Doxa Cuadernos de Filosofía del Derecho, No 40, 2017, p. 89.

45 Para el significado de factum probandum y de otra terminología del derecho probatorio ver el glosario de Anderson, Terence; Schum, David y Twining, William, Análisis de la prueba, Marcial Pons, Madrid, 2015, pp. 445-451.

${ }^{46}$ El papel de la coherencia narrativa es resaltado por el modelo del story model que resalta al holismo como perspectiva de análisis propia del razonamiento lego sobre los hechos. Dicho modelo se vincula con teorías de psicología cognitiva y ha sido utilizado, especialmente, para dar cuenta del razonamiento del jurado. Frente a aquella se erigen teorías atomistas que enfatizan un tipo de conexión estrecha entre la conjetura a probar y las pruebas disponibles en el caso concreto, las que se resisten a un análisis desde la pura coherencia del relato presentado (el mundo de los científicos pareciera operar de trasfondo). Lo que primaldebiese primar, probablemente, llama a poner atención a ambas perspectivas. AcCATino, Daniela, "La arquitectura de la motivación de las premisas fácticas de las sentencias judiciales”, en PÁEZ, Andrés, Hechos, evidencia y estándares de prueba, Universidad de los Andes, Bogotá, 2015, pp. 70 y ss.

${ }^{47}$ Coloma, Rodrigo, "Las buenas y las malas historias. Criterios de validación del discurso de los hechos en las sentencias judiciales”, en Brunet, Pierre; Arena, Federico (dirs.), Cuestiones contemporáneas de teoría analítica del derecho, Marcial Pons, Madrid, 2011, pp. 87-93. 
(c) una generalización es acertada si me ha sido útil en el pasado ${ }^{48}$; (d) los niños no mienten ni pueden ser influenciados por los adultos o, (e) los peritos no se equivocan, porque son expertos en su disciplina ${ }^{49}$.

(c) Reconocimiento de restricciones probatorias desde la ética profesional. Los abogados probamos hechos usando reglas institucionales y éticas que pueden impedir acceder a la verdad, ganar un litigio o condenar a un culpable. La comunidad jurídica valora el secreto profesional, la sujeción a la voluntad del cliente y la evitación de la victimización por sobre el resultado de las gestiones judiciales. Apegarse a estas reglas genera efectos que son muchas veces incomprensibles para los legos. Fuera de la comunidad jurídica, la deontología no es una razón sólida para evitar ciertos resultados. El obstáculo epistémico implicado en la visión del lego puede describirse como la creencia de que la evaluación del propio desempeño solo está determinada por el resultado del litigio. Este obstáculo genera dos errores: (i) desatención de las reglas éticas y (ii) desatención de los intereses del cliente ${ }^{50}$.

(d) Utilización de un estándar de prueba. Un componente que genera perplejidades al argumentar y decidir hechos es el uso de un estándar de prueba. Aun cuando lo aplicamos también en la vida diaria, nos resulta difícil aceptar que cuando decidimos hechos asumimos diferentes riesgos en orden a que podemos estar equivocados. El obstáculo epistémico en lo que a estándar de prueba incumbe se relaciona con la negación a hacer visibles las posibilidades de error, cuando se asocian a los hechos probados determinados efectos prácticos. De la mano de ello se encuentra el obstáculo epistémico que apunta a no diferenciar los riesgos permitidos dependiendo de la decisión de la que se trate.

(e) Fundamentación de la decisión de los hechos probados. Una actividad que da cuenta de lo precedentemente señalado, pero que a la vez presenta dificultades propias, es la construcción del discurso que justifica la decisión en las sentencias. Así, al fundamentar las sentencias se hacen visibles nuestras concepciones respecto de los hechos probados, nuestras faltas de rigor analítico al valorar la prueba, etcétera. El texto esperable debe estar estructurado de manera autónoma (no presupone saberes especializados), profunda (debe decir más de aquello que un observador medianamente agudo podría extraer de una lectura superficial) y, a la vez, debe ser económico (debe limitarse a lo que argumentativamente conecta disposiciones normativas y las pruebas rendidas).

48 Arena, Federico, "Los estereotipos normativos en la decisión judicial: Una exploración conceptual", en Revista de derecho Universidad Austral de Chile, vol.29, Nº 1, 2016, pp. 51-75.

${ }^{49}$ Como un ejemplo de uso de estos saberes folk pueden entenderse las críticas a las razones que David Hume tiene en cuenta para atribuir credibilidad a las declaraciones de testigos (PÁEZ, Andrés, "Una aproximación al testimonio como evidencia", en VÁsQuez, Carmen (ed.), Estándares de prueba y prueba científica. Ensayos de epistemología jurídica, Marcial Pons Madrid, 2013, pp. 218-219). Hume, a su vez, las intentaba combatir con otros saberes folk que eran esgrimidos en su época Cfr. Hume, David, Investigación sobre el conocimiento humano, Alianza, Madrid, 2012, pp. 147-173.

${ }^{50}$ Un estudiante debiera tener en consideración, por ejemplo, el impacto provocado por la renuncia de la víctima, en sede penal, a una indemnización potencialmente cuantiosa. Ver Ministerio Público con Jobn Joseph Reilly, sentencia del Tercer Tribunal del Juicio Oral en lo Penal de Santiago, de 11 de noviembre de 2014, RIT $\mathrm{N}^{\circ} 159-2014$. 


\section{El uso de PROBlemas EN UN CURSO DE DERECHO PROBATORIO. UN EJEMPLO}

Lo anterior será ilustrado mediante un ejemplo extraído de un curso comprometido con el desarrollo de la competencia "resuelve conflictos jurídicos" y cuyos participantes son estudiantes en la fase final de su proceso formativo. Usaremos un examen final escogido al azar en que se retroalimenta al estudiante acerca de los obstáculos epistémicos que dificultaron un mejor uso de los recursos probatorios disponibles ${ }^{51}$.

a) El problema presentado. Se pidió adoptar la posición de un abogado que representa a la familia de George Mallory, ya sea ante las autoridades administrativas o judiciales (los estudiantes debían optar). Un punto clave para el éxito o fracaso de la solicitud dependía de la prueba de si George Mallory ${ }^{52}$ alcanzó la cima del Everest en la expedición que comandó durante 1924. Para estos efectos los estudiantes leyeron 18 páginas con información diversa que podía ser utilizada como prueba indirecta o circunstancial de la llegada a la cumbre. La actividad más importante era la siguiente: "Construya un escrito conducente a obtener que la estatua conmemorativa de la ascensión al Everest represente a George Mallory I, como asimismo que sus descendientes sean invitados a la inauguración del monumento".

b) Fragmento de la respuesta de un estudiante: "Para estos efectos se encuentra el testimonio de un alpinista quien acompañó en la campaña a Mallory, el descubrimiento del cuerpo de dicho alpinista que hasta hace poco estaba perdido en la montaña y que dada las condiciones ambientales se encontraba bien conservado, inclusive encontrándose parte de su vestimenta en buen estado, y que constata la posibilidad de soportar las inclemencias de la alta montaña. Se encuentra también los resultados de las pruebas para sobrevivir en condiciones como las de la montaña en donde Mallory es catalogado excepcional llegando casi al máximo de puntaje. Hay declaraciones de peritos dedicados a la montaña quienes dedicados a la búsqueda del desaparecido cuerpo, pueden evidenciar que hay serias sospechas que George hizo cumbre y murió mientras este descendía.

A mayor abundamiento hay pruebas que certifican que la vestimenta usada era eficaz para soportar el clima de montaña”.

c) Fragmento de la corrección realizada por el profesor: "El texto construido es muy débil. No aprovecha el potencial explicativo de los recursos probatorios disponibles. Respecto de los que elige no construye relaciones estrechas con lo que se trata de demostrar". Con esta retroalimentación se comunica que no se ha ido lo suficientemente lejos en el razonamiento

${ }^{51}$ El examen identificado con el $\mathrm{N}^{\circ} 28$ obtuvo una calificación de 3,8 sobre 7,0 (la nota de aprobación era 4,0). Fue tomado el 2010. La locución obstáculo epistémico no fue usada explícitamente en el curso correspondiente (Argumentación y Análisis Jurisprudencial de la carrera de derecho de la Universidad Alberto Hurtado). Los responsables de la asignatura eran los profesores Flavia Carbonell y Rodrigo Coloma, siendo este último el autor del problema del que debían hacerse cargo.

52 El problema está construido a partir de un hecho real. Los insumos utilizados fueron extraídos de distintos sitios de Internet en los que se reconstruyen testimonios de algunos acompañantes de Mallory que lograron regresar de la expedición. Asimismo, se dieron indicaciones de las dificultades que, en ese entonces, representaba el ascenso al Everest (incluidas algunas fotos de la ruta seguida). Algunas de las ideas respecto de la manera de presentar las condiciones físicas extraordinarias de Mallory (y de su compañero de escalada final Andrew Irvine) fueron extraídas del libro de Archer, Jeffrey, La senda de la gloria, Grijalbo, Barcelona, 2010. 
probatorio, según era posible al considerar las pruebas disponibles. En otras palabras, mediante un texto bien elaborado era posible articular ciertos puntos que quedaban muy abiertos de la lectura del problema presentado. Tanto el abogado como el juez prolijo deben realizar un esfuerzo relevante para la construcción de trayectorias argumentativas que exige una decisión que da por probados hechos discutidos por las partes en un proceso judicial ${ }^{53}$. Esto se verá con un poco más de detalle en la letra a continuación.

d) Obstáculos epistémicos. En primer lugar, la respuesta usa un estándar de prueba folk. Este obstáculo epistémico lo lleva a cometer un error: el estudiante no explicita el estándar de prueba que utiliza para dar por probado el hecho discutido (alpinista hizo cumbre $)^{54}$. En segundo lugar, la respuesta asigna la misma relevancia a todos los medios de prueba. Este obstáculo epistémico se expresa en una omisión; no hace mención al problema de la gradualidad de la relevancia de los medios de prueba ${ }^{55}$. En tercer lugar, la respuesta del estudiante es la de un principiante no experto. Al no tener claridad respecto de cuál es la dimensión normativa del problema (la que no es cuestionada) y al intentar resolver la tarea usando el sentido común, el estudiante incurre en el error de confundir dos asuntos diferentes; si el alpinista hizo (o no) cumbre (probatorio) y si la familia del alpinista tiene (o no) derecho reclamar honores (jurídico). En cuarto lugar, la respuesta no supera el obstáculo epistémico acerca de la accesibilidad del pasado. Confía en que un hecho ocurrido hace casi cien años admite ser reconstruido de forma similar a un hecho reciente. Este obstáculo se expresa en un error; la respuesta no se hace cargo del paso del tiempo entre el hecho a probar y el momento en que la prueba se rinde. De ese modo, no anticipa posibles objeciones como datos perdidos, imposibilidad de acceso a ellos o baja confiabilidad de los datos obtenidos. En quinto lugar, el uso de la narrativa como herramienta para conectar enunciados es bastante precaria, habida cuenta de lo que trata de ser demostrado. Así, carece de sutilezas en orden a reducir los saltos argumentativos ${ }^{56}$. A contrario, se conforma con enumerar algunas piezas probatorias esperando que sea el lector quien deba hacer la tarea de establecer conexiones

${ }^{53}$ El estudiante debiese asumir un mayor esfuerzo en la tarea realizada: no operar desde el sentido común o desde aquello que le resulta intuitivo. Los psicólogos y neurocientíficos han abordado el asunto distinguiendo dos sistemas de pensamiento; automático y esforzado. Ambos son muy útiles dependiendo de la tarea que se trate de abordar. En los problemas probatorios, hay buenas razones para concluir que un pensamiento muy automatizado favorece los errores. Para la distinción entre ambos sistemas, Kahneman, Daniel, Pensar rápido, pensar despacio, $1^{\mathrm{a}}$ edición, Debate, Buenos Aires, 2012.

${ }^{54} \mathrm{La}$ frase ... hay serias sospechas que George hizo cumbre es clave para sustentar este juicio evaluativo. La respuesta refleja que no se resuelve el problema con estándares artificiales como el estándar de prueba penal u otro análogo. Evidencia de aquello es el uso de lenguaje cotidiano para formular la respuesta y la presencia de debilitadores discursivos (serias sospechas) en ella.

${ }^{55}$ Si se considera la conjetura que estaba tratando de ser demostrada, un buen razonamiento, tiene que dar cuenta de que algunas de las pruebas mencionadas cumplen un papel mucho más significativo que otras.

56 Chesterton habla de que en un relato de misterio aquello que nos provoca perplejidad "no es lo inexplicable, sino la explicación que todavía no hemos oído. Eso que llamamos arte o progreso: el avance de lo complejo hacia lo simple”. Chesterton, G. K. “Civilización y progreso”, en Chesterton, G. K. Cómo escribir relatos policiacos. Acantilado, Barcelona, 2011, pp. 34-35. En las sentencias judiciales el asunto no es muy distinto, el lector busca las conexiones que de alguna manera le hagan sentir que el relato presentado es apto para dar sentido a todo aquello que probatoriamente ha ocurrido. 
con la conjetura debatida. En pocas palabras, hay un escaso esfuerzo argumentativo que es promovido, tal vez, por un erróneo punto de partida que podría irónicamente reconstruirse como sigue: ;cualquier intérprete medianamente agudo debiera ser capaz de inferir tal conclusión desde los datos disponibles: si no lo visualizas, el problema no es mío!

El modo folk de resolver problemas de prueba es excesivamente optimista y confiado en las generalizaciones que confirman la hipótesis que pretendemos probar. El obstáculo epistémico es, de alguna forma, la tendencia a usar el modo en que cotidianamente valoramos la prueba en contextos en donde las instituciones nos fuerzan a usar patrones de valoración y de argumentación más elevados. En la situación presentada solo hay prueba circunstancial y, entonces, quien no conoce el modo estandarizado de sortear el problema no puede evitar incurrir en saltos de razonamiento adecuados para la vida diaria, pero injustificados en el marco de la tarea requerida. Si el estudiante no conoce un procedimiento de análisis de evidencias (por ejemplo, chart method ${ }^{57}$ ) le resultará imposible superar la tarea correctamente, es decir, construir un texto que deje claro el uso de un razonamiento sofisticado al valorar la prueba disponible. En este sentido, el problema presentado es un buen problema jurídico, pues exige dominar las estrategias de razonamiento implicadas en el aprendizaje del derecho probatorio.

\section{CONCLUSIONES}

El derecho probatorio es un marco de restricciones al sentido común que intenta asegurar la racionalidad. El entramado de disposiciones respecto de la sana crítica, los medios de prueba, el estándar de prueba, el resultado probatorio, la justificación de la decisión y otras tantas dispersas en el ordenamiento, pretenden controlar las preferencias del adjudicador y restringir el modo en que toma una decisión. Pero como sabemos todos los abogados, el conjunto de estas restricciones no determina todo el proceso de razonamiento y de decisión, ya que existen amplios sectores de la prueba de los hechos por donde se cuelan las intuiciones, las preferencias infundadas, los prejuicios y los estereotipos.

El aprendizaje del derecho probatorio es estratégico, es decir, orientado a la toma de una decisión jurídicamente óptima (i.e. racional). Este modo de entender el derecho probatorio implica que el aprendizaje central del estudiante no es instruirse en el listado de limitaciones, sino que en el conjunto de técnicas de razonamiento y de toma de decisiones que son válidas dentro de ese marco de restricciones y que es aplicable a un amplio número de casos en donde existe un umbral de incertidumbre acerca de los hechos.

Este modo de entender la disciplina y su aprendizaje distingue al curso de derecho probatorio de los cursos dogmáticos, de destrezas de litigación y de teoría de la argumentación. Además, permite sostener que el curso de derecho probatorio debe configurarse sobre

${ }^{57}$ En la asignatura se enseñó el Chart Method de Wigmore, con adaptaciones. Concerniente a este método, además de los textos fundacionales de Wigmore, de las adaptaciones de TwinING, Schum y otros, ver DeL VAlle, Carlos y AgüEro, Claudio et al., "Aproximación al análisis de la valoración de la prueba usando modified wigmorean analysis (MWA)”, en Ius et Praxis, vol.15, $\mathrm{N}^{\circ} 1$, pp. 15-53. 
la base de "problemas jurídicos", en cuanto recurso didáctico clave para identificar y superar los obstáculos epistémicos típicos que el razonamiento probatorio presenta.

Un buen problema jurídico solo puede ser resuelto si el estudiante domina una o más competencias relevantes. La famosa frase de Bachelard El hombre culto es como un zurdo obligado a usar la mano derecha pone énfasis en este punto. La función de los problemas es forzar a los estudiantes a pensar de un modo que es ajeno a la vida cotidiana. Si el estudiante no despliega la competencia esperada, el problema hace visibles los obstáculos epistémicos que impidieron resolverlo con éxito; así, los errores son síntomas de esos obstáculos. El rol del profesor es identificar errores, diagnosticar obstáculos y ayudar a los estudiantes a superarlos.

El concepto de "obstáculo epistémico" desplaza la idea de que el estudiante es una tabula rasa para el aprendizaje. Todo estudiante ha resuelto problemas de prueba en su vida diaria y conoce la heurística que le ha permitido ofrecerles una solución. El asunto es que en contextos especializados como un procedimiento judicial esa heurística no sirve, es necesario deconstruirla y aprender aquella que usan los abogados y jueces experimentados.

\section{BiBLIOGRAFÍA}

Accatino, Daniela, 2015: "La arquitectura de la motivación de las premisas fácticas de las sentencias judiciales”, en Páez, Andrés, Hechos, evidencia y estándares de prueba, Universidad de los Andes, Bogotá, pp. 65-87.

AgÜERo San Juan, Claudio, 2014: "Una crítica al modelo de la narración judicial como explicación científica", Ius et Praxis, vol.20, n.1, pp. 221-252.

AgüEro San Juan, Claudio, 2018: "La Sana Crítica como cláusula general”, en Benfeld, Johann y Larroucau, Jorge (editores), La sana crítica bajo sospecha, publicaciones de la Escuela de Derecho PUCV, Valparaíso, p. 153-162.

Anderson, Terence; Schum, David y Twining, William, 2015: Análisis de la prueba, Marcial Pons, Madrid.

ArCher, Jeffrey, 2010: La senda de la gloria, Grijalbo, Barcelona.

Arena, Federico, 2016: "Los estereotipos normativos en la decisión judicial: Una exploración conceptual”, en Revista de Derecho Universidad Austral de Chile, vol. 29, Nº 1, pp. 51-75.

Astolfi, Jean Pierry y Peterfalvi, Brigitte, 1997: "Estrategias para trabajar los obstáculos: dispositivos y resortes”, en Camilloni, Alicia (comp), Los obstáculos epistemológicos en la enseñanza, Gedisa, Barcelona, 224 páginas.

Astolfi, Jean Pierry, 1994: "El trabajo didáctico de los obstáculos, en el corazón de los aprendizajes científicos”, en Enseñanza de las Ciencias, vol. 12, N 2, pp. 206-216.

Astolfi, Jean Pierry, 2011: El error un medio para enseñar, Díada, Sevilla, 100 páginas.

BACHELARD, Gastón, La formación del espíritu científico: contribución a un psicoanálisis del conocimiento objetivo, Siglo XXI, Madrid,

Brousseau, G., 1998: Théorie des situations didactiques, La Pensée Sauvage, Grenoble.

BurRIDGE, Roger, 2002: "Learning law and legal expertise by experience", en Burridge, Roger et al., Effective learning $\mathcal{E}$ teaching, The Institute for Learning and Teaching in Higher Education, Londres, pp. 25-51.

Carlino, P., 2013: “Alfabetización académica diez años después”, en Revista Mexicana de Investigación Educativa, vol. 18 (57).

Coloma, Rodrigo, 2017: "Bases conceptuales de un modelo para decidir hechos", en Doxa Cuadernos de Filosofía del Derecho, No 40, pp. 69-92. 
Coloma, Rodrigo, 2009: "Estándares de prueba y juicios por violaciones a los derechos humanos", en Revista de Derecho Universidad Austral de Chile, vol. XXII, Nº 2.

Coloma, Rodrigo, 2011: "Las buenas y las malas historias. Criterios de validación del discurso de los hechos en las sentencias judiciales", en Brunet, Pierre; Arena, Federico (dirs.), Cuestiones contemporáneas de teoría analítica del derecho, Marcial Pons, Madrid.

Coloma, Rodrigo, 2016: "Las disciplinas jurídicas y su reinvención”, en Ius et Praxis, año 22, $\mathrm{N}^{\circ}$ 2, pp. 253-298.

Del Valle, Carlos y AgüEro, Claudio et al., 2009: “Aproximación al análisis de la valoración de la prueba usando modified wigmorean analysis (MWA)”, en Ius et Praxis, vol.15, No 1, pp. 15-53.

Ferrer, Jordi, 2007: La valoración racional de la prueba, Marcial Pons, Madrid.

Festinger, León, 1975: Teoría de la disonancia cognoscitiva, Instituto de Estudios Políticos, Madrid. Figueroa, Gonzalo, 1988: Curso de Derecho Civil: Materiales para clases activas. Teoría de la ley y los derechos subjetivos. $2^{\mathrm{a}}$ ed. Santiago, Editorial Jurídica de Chile.

García Petit, María Cecilia y Agüero San Juan, Claudio, 2014: "Bases para el estudio de la dinámica discursiva en la comunidad jurídica chilena”. Revista de Derecho (Valdivia). vol. 27, n. 1, pp. 59-79.

GonZÁlez Lagier, Daniel, 2018: "Distinciones, estipulaciones y sospechas sobre los criterios de valoración y los estándares de prueba”, en Benfeld, Johann y Larroucau, Jorge (eds.), La sana crítica bajo sospecha, Ediciones Universitarias de Valparaíso, Valparaíso, pp. 23-24.

HernÁndez, Juan, 2012: Sociología de las profesiones sanitarias, Escuela Nacional de Sanidad, Madrid. Hume, David, 2012: Investigación sobre el conocimiento bumano, Alianza, Madrid.

Kahneman, Daniel, 2012: Pensar rápido, pensar despacio, $1^{a}$ edición, Debate, Buenos Aires.

LaUdan, Larry, 2005: "Por qué un estándar de prueba subjetivo y ambiguo no es un estándar", en Doxa Cuadernos de Filosofía del Derecho, $\mathrm{N}^{\circ} 28$.

LuCARELLI, F., 1999: "Teoría y práctica como innovación en docencia, investigación y actualización pedagógica”, en Cuadernos de Investigación, No 10, Instituto de Ciencias de la Educación, Buenos Aires, Facultad de Filosofía y Letras, UBA.

Marzábal, Ainoa; Merino, Cristián; Rocha, Alejandro, 2013: "El obstáculo epistemológico como objeto de reflexión para la activación del cambio didáctico en docentes de ciencias en ejercicio", en Revista electrónica de investigación en educación en ciencias REIEC, vol. 9. $\mathrm{N}^{\circ} 1$, pp. $70-83$.

Mora, Arabela, "Obstáculos epistemológicos que afectan el proceso de construcción de conceptos del área de ciencias en niños de edad escolar", en InterSedes, Revista de las Sedes regionales, Universidad de Costa Rica, vol. 3, No 5, mayo 2002, pp. 75-89.

PÁEz, Andrés, 2013: “Una aproximación al testimonio como evidencia”, en Vásquez, Carmen (ed.), Estándares de prueba y prueba científica. Ensayos de epistemología jurídica, Marcial Pons Madrid.

PARK, R., SAKs, M., 2006: "Evidence Scholarship Reconsidered: Results of the Interdisciplinary Turn", en 47 Boston College Law Review.

Tversky, Amos y Kahneman, Daniel, 1974: "Judgment Under Uncertainty: Heuristics and Biases", en Science, vol. 185.

Tversky, Amos y Kahneman, Daniel, 2012: "El juicio bajo incertidumbre: heurísticas y sesgos”, en Kahneman, Daniel, Pensar rápido, pensar despacio, Debate, Buenos Aires: Debate, pp. 545-567.

White, Aidan, "Información falsa: La opinión de los periodistas", en Correo de la Unesco. Disponible en "https://es.unesco.org/courier/2017-julio-septiembre/informacion-falsa-opinion-periodistas" [última visita: 16 de mayo 2018].

William, Leonard; Gerace, William y Dufresne, Robert, 2002: "Resolución de problemas basada en el análisis. Hacer del análisis y del razonamiento el foco de la enseñanza de la física", en Enseñanza de las Ciencias, No 20 (3), pp. 387-400. 